\title{
Self-Learning Control System Concept for APU Test Cells
}

\author{
Razvan Ciobanu ${ }^{1 *}$, Adrian Stoicescu ${ }^{2}$, Cristian Nechifor ${ }^{2}$, and Alexandra Taranu ${ }^{1}$ \\ ${ }^{1}$ COMOTI - Romanian Research \& Development Institute for Gas Turbines, Metrology Laboratory 061126 Bucharest, Romania \\ ${ }^{2}$ COMOTI - Romanian Research \& Development Institute for Gas Turbines, Automation Department 061126 Bucharest, Romania
}

\begin{abstract}
The proposed concept presents an innovative test cell control system, compatible with an existing APU (Auxiliary Power Unit) test cell. The system is essentially a Non-Propulsive Energy (NPE) Power Management Unit that needs to efficiently distribute power among an aircraft's pneumatic and electrical loads, based on key parameters read from: loads (electrical, pneumatical), a real APU and realtime models of main engines representative to the aircraft. For this, the concept suggests a hardware \& software solution, based on the approach of Artificial Neural Network (ANN). The ANN processes all inputs according to a mathematical law trained from existing data sets, such that minimal power loss is considered, given all safety levels are achieved. Development of the neural network is made such that the fastest response time and best performance consist as general goals, and the resulting control system is tested via Hardware-in-the-Loop simulation. Thus, the neural network is also designed to be safe and stable given maximum performance. The hardware solution describes all the equipment included to fulfil the objectives of the concept.
\end{abstract}

\section{Introduction}

Given the technological development in the past 10 years with regard to on board power management units (PMU's) it can be clearly seen that a necessity for adaptive control power management units had up risen. Power management on an aircraft is critical regardless of its dimension or of its nature (either fixed wing or rotorcraft). Given the fact that the aerospace and aeronautical fields are searching for means of reducing emissions and thus, fuel (energy) consumption, the onboard power management units type LRU's (Line Replaceable Units) are going through substantial system changes. These changes can account for systems such as:

- Electrical energy management of critical, essential and nonessential systems

- Hydraulic power management of actuators for control surfaces and other type of hydraulic based systems

- Pneumatic power management for systems such as HVAC (Heat Ventilation and Air Conditioning)

These three main categories can account for a total loss of energy of up to $15 \%$ on the basis of overshooting of control signals, less adaptive control systems and a far wider margin of power reserve to adapt for.

For instance, an aircraft's APU (Auxiliary Power Unit) can take up to five minutes to reach an optimal idle regime. This slow start is mainly caused by simplified control systems that only provide a fixed value for the APU's gas generator turbine rpm [1]. Also due to high gas dynamics and inertia, APU's are susceptible to rapid acceleration if fuel dosage and flow is not adaptively controlled [2]. As a consequence, to a slow starting APU, there is a significant amount of burnt fuel. This fuel quantity can be drastically reduced if the APU's electronic control unit (ECU) has implemented in it's controller a fast start ramp with the addition of a Kalman based filter to predict rpm rise and avoid overshot [3]. The disadvantage is that in case of very short rpm rise time periods, a Kalman based limitation filter is inadequate and limited in accurately predicting value rise. Such that for this research topic, a neural networkbased control system was approached.

The conventional aircraft propulsive system uses a combination of hydraulic, electric, pneumatic and mechanical power transfer systems. Increasing use of intelligent control is seen as a direction for advanced aircraft power systems based on rapidly evolving technology advancements in power electronics, faulttolerant electrical power distribution systems and electric driven primary flight control actuator systems [4]. The power used in normal flight conditions by the entire aircraft is generated by AC generators driven by the main engines. During ground maintenance and for the engine start, the power is provided by the APU. Most aircrafts can use the APU during normal flight conditions as backup power sources, in case of engine generators failure. The APU includes a constant speed generator with a three-phase output.

Artificial neural networks (ANN) are computational models inspired by the biological neural networks in animal brains. These systems learn progressively to perform tasks by training from examples rather than explicitly programmed. The training is done within offline computer simulation program that uses a large

* Corresponding author: razvan.ciobanu@,comoti.ro 
number of input-output example patterns [2]. The ANN is based on a collection of connected units called artificial neurons, which are connected in layers. Signals travel from the first (input), to the last (output) layer. The aim of the neural network into this concept is to create an adaptive control loop for the system's Power Management Unit (PMU).

One of the ambitions of the concept is that we would collect data such as pressure and temperature along the pneumatic circuit of the APU Test Cell, to identify and determine the nature of all pneumatic energy loss in order to optimize the energy consumption of the APU. In order to achieve that, we use electronic pressure transducers to determine line pressure level and pressure loss during operation, and thermosensitive probes (RTD) to determine compressibility, thermodynamic parameters of the air/gaseous media in the circuit.

Given the fact that throughout the years there have been many ideas and research carried out in the branch of neural network-based controllers design, we have the ambition of accumulating all knowledge and implementing it in a specific artificial intelligence-based controller. This will create a starting point for all artificial intelligence-based controllers with wide applications in aviation. Possibilities are endless regarding neural networks given the computational power at hand nowadays.

Based on preliminary results further showed, the proposed system is expected provide the environment to explore and validate the integration of fuel efficient propulsion concepts for next generation aircraft (including More Electric Aircraft), via an extensive hardware-software solution. Additional percentages of fuel burn for the CROR (Counter-Rotating Open Rotor) and UHPE (Ultra High Propulsion Efficiency) concepts are obtained by implementing control strategies described further in this section, driving the machines beyond their standard regime levels.

This paper presents the envisioned system, with both hardware and software considerations at the basis of its novelty. The main testing method is presented, as well as preliminary results that point to the level of achievement in developing the experimental stage of the concept.

\section{Neural networks in aerospace applications}

Up until the late ' $70 \mathrm{~s}$, ANNs were purely theoretical concepts given computational power of early computers and processors. The first military application of neural networks was implemented by the USAF in the 1980's, in the long-range Tomahawk missile guidance and image acquisition sensor. The first civilian applications in aviation were developed around the year 1985 in USA as a replacement for conventional PID and Kalman filterbased algorithms for autopilots. These applications were only tested experimentally in medium size test aircraft. In these test aircraft the yaw damper and the autopilots pitch channel were modified with a neural network and gave impressive results given normal atmospheric conditions.
Due to low computational power back in the early 1980 's the research of implementing artificial neural networks in aircrafts was abandoned. Later research was carried on by many universities regarding implementation of artificial intelligence in aircraft with significant increase in knowledge of behaviour of neural networks in airplane dynamics and control systems. Individual studies were carried out by many students, engineers and Ph.D.'s in the past couple of years with substantial increase in optimization and implementation in small, minimal UAVs/RPAS and drones alike. These studies were only concluded by small demonstrators with a low level of implementation of neural networks due to lack of funds.

In USA, NASA Dryden Flight Research Centre launched a project to exploit revolutionary technologies in flight control that can optimize aircraft performance in both normal and failure conditions. Their project was carried out from the beginning of the year 1996 until the year 2002 with the name Intelligent Flight Control System (IFCS) and was implemented on an F-15 type fighter jet [3].

From the Georgia Institute of Technology, School of Aerospace Engineering [4] we can say that they have made a significant leap toward nonlinear adaptive flight control using neural networks. As a conclusion towards their work, the boundedness of tracking error and control signals is guaranteed, the architecture can accommodate both linear in the parameters networks as well as single or multi-layer perceptron neural networks.

In thesis [5] submitted to the faculty of the Virginia Polytechnic Institute and State University it is shown that the energy balance concept in combination with a neural network was successfully developed and applied to modelling aircraft energy performance. The energy balance algorithm accepts as inputs aircraft specific limitations, such as velocity, altitude, and weight. Other equations compensate for altitude change giving both minimum and maximum conditions. When the performance data is generated it directly goes into the neural net as input vectors and target vectors. Two inputs containing 600 randomly generated pieces of data flow feedforward and then are backpropagated until the net reaches its sum-square error goal. In [6] research was made in the direction of fault detection and isolation regarding gas turbine engines. Their work showed results such as approach by using multiple model or bank of parallel dynamic neural networks for proposing fault detection and isolation schemes. One can conclude that the two parameters, namely N2 and PLC have good monitoring capabilities with respect to the common faults that can occur in the aircraft jet engines. Dynamic neural networks have also recently been exploited and employed in achieving fault detection and isolation of nonlinear systems [6-9]

The study of neural networks is at its highest since the year 2010 and we consider that sufficient time has passed since it was last tested in aviation demonstrators, and we confidently say that it will create a new leap in aviation ethics and design criteria. 


\section{System concept and architecture}

The concept system (further designated "APRIL" Aircraft Power Regulation Integrated Logic) is designed be integrated into an existing facility that should include an APU Test Cell, with a real APU (turbine, startergenerator), a pneumatic circuit and electrical loads, as seen in Fig. 1. The Main Engine Models are provided to APRIL from an external source. The APU Control Room is the user operating location of the Test Cell and includes the PMU and its enclosure, various instrumentation adapters, relays, switches, supplies, etc. In order to discuss the elements and operation of APRIL within the facility, a few related systems need to be discussed.

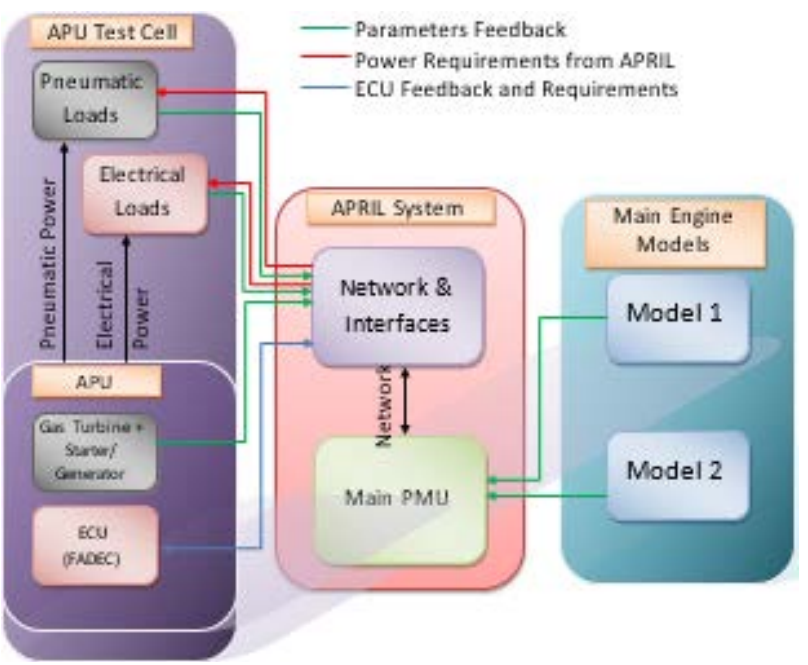

Fig. 1. APRIL power sharing architecture.

The pneumatic system of the APU is contoured around a twin-engine design, capable of providing engine start sequence and de-icing capabilities for both engine cowlings and wing leading edges. It provides low pressure (LP) air for the engine start sequence, one at a time through the means of the installed bleed valves and isolation valves; e.g. if we chose to start the left hand (LH) engine, the cross-feed isolation valve (central position) will close and the high pressure (HP) bleed valve on the engine will also close; thus, providing start air through the LP starter valve. A real-time data acquisition of the pneumatic system shall provide valuable information for monitoring the overall aspect of the behaviour of the PMU software.

The electrical network consists of two engine-driven generators (simulated) and one APU generator (real). $\mathrm{AC} / \mathrm{DC}$ rectifiers are used to convert $\mathrm{AC}$ voltage with fixed frequency at the main $\mathrm{AC}$ bus to multi-level DC voltages at the secondary buses which supply electrical power to loads.

Overall, the APRIL includes both the main PMU, which is a computing system that efficiently distributes power from the APU to pneumatic and electrical loads.
This is done based on key parameters inputs received, via local communication network and interfaces, from the loads, from two main engine models (simulated) and from specific aircraft mission profiles data. The algorithms for the power distribution are run by the PMU, which in turn is in charge of controlling the APU through its ECU (Engine Control Unit). The PMU is programmed with a self-adapting control loop that allows testing various scenarios.

From the hardware point of view, the PMU is configured based on the resources needed to run smoothly up to seven hidden layer Neural Network control loops, being equipped with an 8-core embedded controller. It also needs to interface with all the types of input/output signals existing in the Test Cell, relevant to the existence and purpose of the system.

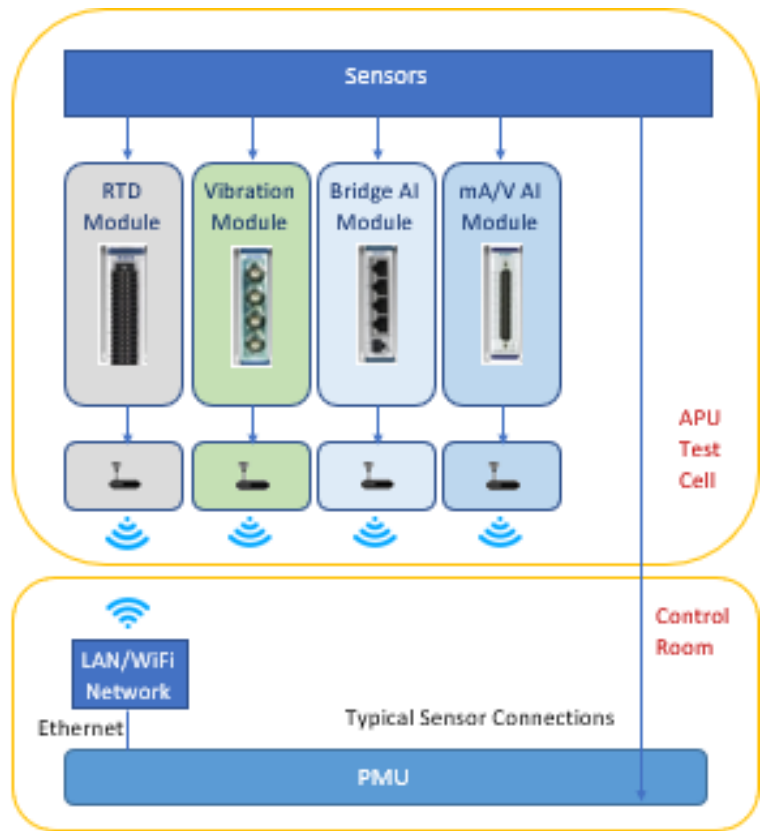

Fig. 2. Wireless instrumentation nodes.

In terms of wiring efficiency, both at ground and in air, it is desirable to have local group of sensors connected together in certain parts of the aircraft or (in this case) Test Cell, that send data wirelessly via common (joint) instrumentation nodes. A proposed architecture is shown in Fig. 2, which is based around four local wireless transmission modules. Each module can receive input from multiple sensors or transducers of similar type and send data across the facility, between the APU Test Cell and the Ethernet/Wi-Fi network in the APU Control Room. Advantages of this architecture include: improved electrical protection, ATEX protection, optimized wiring solutions for sensors in different parts of the Cell (similar to aerospace situations). Four modules were considered, each one being integrated into a compact chassis with Ethernet and Wireless capabilities. This instrumentation node solution provides performance in terms of parameter resolution (up to 24-bit) and sample rates higher than a typical PLC system. For innovative miniature sensors, low-power instrumentation nodes may be taken into consideration, such as I2C digital microprocessor 
sensors, powered by batteries or alternative energy harvesters.

\section{Control loops}

The aim of the neural network into this project is to create an adaptive control loop for the PMU. The inputs of the neural network will consist of 6 ports $\left(\mathrm{x}_{1}\right.$ to $\left.\mathrm{x}_{6}\right)$, as seen in Fig. 3.

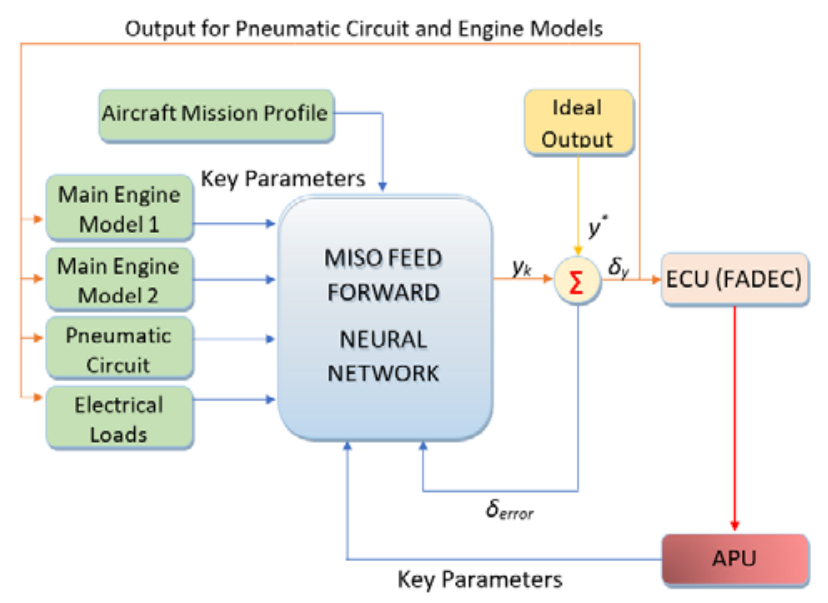

Fig. 3. Neural network basic connection diagram.

Aircraft mission profile power requirements - the minimum power level generated by the APU to maintain all corresponding systems online and in safe working condition without jeopardizing the mission and/or passengers with respect to mission stages.

Main engine models 1 and 2 power requirements the electric power generated by each individual engine to the on-board main power bus such that all electrical needs are achieved. During flight, these parameters may change or drop completely. Thus, the APU must have the capability to achieve a certain level of power to compensate for the drop or loss of energy from the engines.

Pneumatic circuit power requirements - the power consumed by the pneumatic circuit in order to perform specific tasks on the ground or in air by the means of different mission profiles or situations that may come up such as: loss of de-icing capabilities from the engine, flow and pressure compensation due to different atmospheric conditions. The APU must provide enough pneumatic energy to compensate for all the abovementioned factors and variables.

Electrical loads power requirements - the power consumed by the electric loads that must be supplied by the APU given different mission profiles, situations and stages. The APU must be capable of supplying critical loads in order not to risk the mission and/or passengers.

$A P U$ power requirements feedback loop - the level of power that the ECU needs in order to maintain such that all loads and power requirements are met.

The feedback loop for the bias's - given an ideal model, this will train the neural network such that the best level of power will be provided at the networks output given any input situation.

All inputs listed above will have a numerical value attributed from 0 to 100 , corresponding to the power needs at hand, given different stages of flight and/or mission profile with respect to loads behaviour.

The neural network will process all inputs according to a mathematical law developed such that minimal power loss will be considered, given all safety levels are achieved. These laws will be introduced in the neurons $\left(K_{n}{ }^{m}\right)$ of the neural network. The adaptive neuron laws considered so far are logsig (logarithmic and sigmoid) and hardlim (hard limit if/else). All neurons will have a given number of inputs (weights, $W_{n}^{m}$ ) in accordance to the number of neurons per layer and inputs to the neuron layer. Also, every single neuron will have a bias $\left(b_{n}{ }^{m}\right)$ input, so that during training of the neural network, the control laws inside these neurons could be modified for optimization.

The neural network will have a given number of hidden layers $\left(\mathrm{HL}_{\mathrm{n}}\right)$, as seen in Fig. 4 , that will be responsible for computing the master power requirement. It will have a single output that consists of a numerical value corresponding to the power requirement of the APU towards the aircraft (i.e. level of minimal output power on the APU to satisfy for pneumatic and electrical supply requirements (hence MISO - Multiple Input, Single Output). The weights alongside the bias inputs ( $b_{1}$ to $b_{n}$ ) are responsible to adjust the input in the activation function $\left(K_{I}\right.$ to $\left.K_{n}\right)$ of the neurons via a summative comparator. The bias has the role of training the activation function according to the weights input such that delays and overshoots of signals are at a minimum.

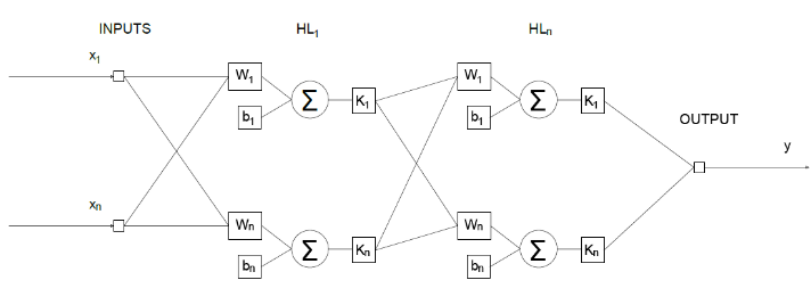

Fig. 4. Neural network layer operation diagram.

An alternative to the artificial intelligence-based controller is a PID based controller. As a backup, we consider the use of this to achieve all command functions of the APU's ECU. The PID has several advantages over the neural network-based controllers such as: small development time span, easy to adjust up to a certain stability level, easy to implement in $\mathrm{C}$ based languages. As a downside we can list: need to implement high order filters (e.g. Kalman) which can significantly slow the controllers' response time, overshoots of signals which can induce system harmonics, long response time in order to achieve good dynamic stability, far less adaptive to a wide span of variance of input signal unlike the neural network. For low signal amplitudes, it has a low signal-to-noise ratio and for high signal amplitudes, it has $90 \%$ of the time an overshot of the signal which will generate delays in response time.

At first, the ANN is developed as a mathematical 
model in order to emulate the needs of the given mission. The development is done in MATLAB and Simulink. This provides a good media for training of the neural network to ensure maximum performance. After development, training and validation in MATLAB Simulink, the neural network is exported as $\mathrm{C}++$ program in order to be implemented in the PMU (NI PXI). The development of the neural network is performed such that the fastest response time and best performance will consist as general goals. Thus, the neural network will also be safe and stable given maximum performance. Training will be performed using different levels of inputs such that we can simulate the behaviour of all systems of the aircraft (i.e. electrical loads, pneumatic circuit, main engines, mission profile etc.). Validation will be done after the above-mentioned goals are achieved and that the neural network is stable. During validation, the following will be considered:

- $\quad$ Random combinations of inputs from all loads will be provided at the neural networks inputs in order to test the response of the neural network;

- Faults will be simulated emulating the loss of one or both starter generators of the aircraft;

- Different mission stages with their own power requirements;

- Different flight profiles which reflect main engine behaviour thus power generation performances;

- Stability in response and minimum overshoots of signals.

\section{Engine models}

The first step in shaping the dynamic model of the engines consisted in determining the thrust developed by them. The following thrust and performance curves were determined: dynamic thrust which was generated by the engine groups during horizontal uniform linear flight, static thrust at the ground, depending on engine speed and air pressure, and the thrust-air density dependence.

For modelling the engine dynamics, we used the following elements: transfer functions, delays, saturation elements and integrators. The purpose of the transfer functions is to define the dynamic response curve of both the execution elements and the aggregates generating gas-dynamic processes. The purpose of the delay elements is to model the mathematical response of the execution elements at various inputs. The saturation elements model the saturation limit of various aggregates, flows, electronic but also mechanical limitations. Integrators define a delay to a square input to the electronic execution elements which consists of an inductive characteristic (e.g. pump motors, starters, spark plugs). The mathematical model was developed deterministically and plays the role of a training set for the neural network to be developed.

However, the main role for training the neural network, in regards to the APU, is played by the APU engine model.

\section{Control system training and simulation}

The APU engine model needs to be compared to the experimental data extracted from the existing APU during operation. After determining the optimum model in terms of accuracy and performance, the final parameter list to be used for training the ANN will be determined. The same steps stand for the main engines, supposing that real engines would be available for testing. However, this application relies on APU testing and performance, implying that main engine characteristics are known. Developing and training a feedforward ANN with higher layers of perceptron`s would help determine characteristic functions of fuel flow for an acceleration curve typical for normal operation. The final controller would be made using the engine models provided and flow functions obtained by means of the neural network.

Neural networks are mainly used for accurate simulation of situations that do not fully allow the definition of a rule set. Their main characteristic is their ability to improve their own performance and adapt to environment changes by learning from experience. Moreover, they are capable of working with noisy or incomplete data sets.

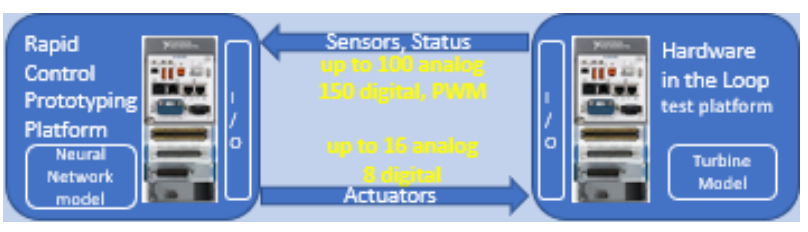

Fig. 5. HIL architecture.

Finally, in order to test and validate the control loops, a real-time Hardware-in-the-Loop (HIL) architecture is proposed, as seen in Fig. 5. During HIL testing, the control system (or controller) interfaces not with any real engine, but with real-time hardware systems running each engine model and simulated loads. These hardware systems are part of a cluster further called "simulator". The simulator outputs generate, based on the neural training previously performed, real outputs of the physical engines and additional systems, and receives control inputs from the controller. This is essentially a closed loop, in which the controlled system condition is received by the controller by means of electric signals similar to engine sensor signals. These parameters are used, via earlier developed control functions, to determine adequate values for reaching desired operation conditions by the most power efficient means.

Generation of stimuli includes generating various engine speed values, environment conditions (e.g. flight conditions) and loads (electrical, pneumatic).

Once the neural training and HIL simulation are complete, the trained logic can be exported and deployed to a less resourceful hardware

The resulting controller is a novel FADEC-type architecture, as illustrated in Fig. 6. 


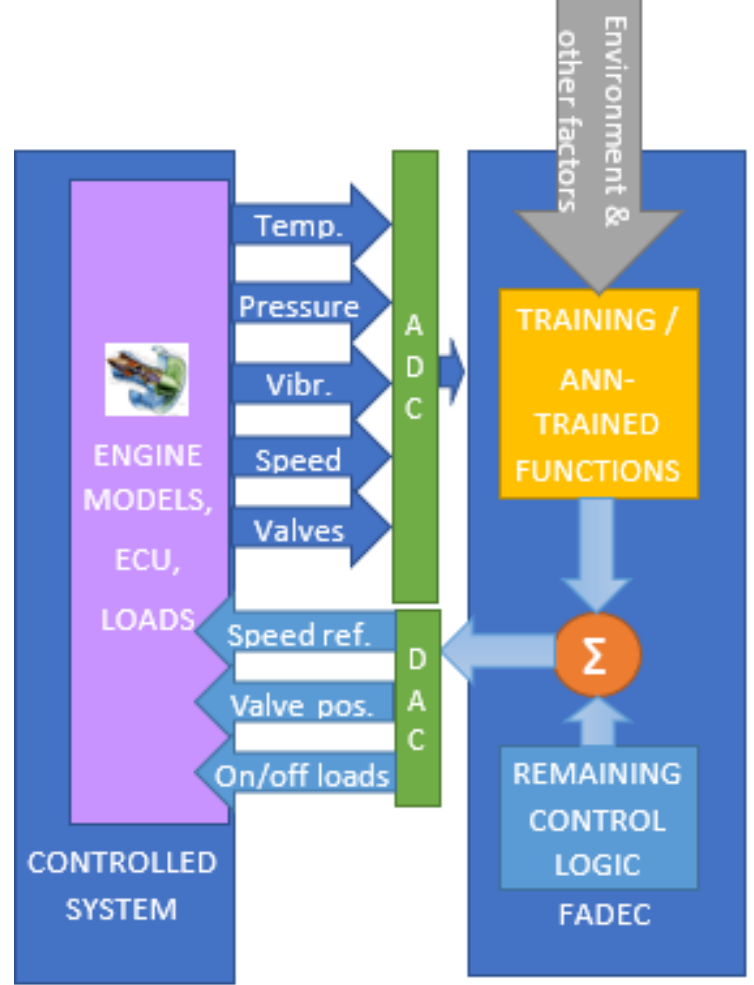

Fig. 6. The novel FADEC principle.

\section{Preliminary results}

Although the whole APRIL concept of control system is yet to be fully tested beyond the concept status, preliminary results showed promising results in regards to ANN self-learning engine control, Hardware-in-theLoop testing for ECUs or fibre optics instrumentation in aerospace applications.

Throughout the latest years, intelligent control methods using fuzzy logic were studied. Other strategies included both fuzzy logic and evolutionary algorithms [10], controlling combustion pressure or other parameters. A similar research was performed in [11] regarding the design and evaluation of two fuzzy controllers by controlling combustion pressure. Even though traditional control methods are well-established and reliable, modern control methods are expected to deliver improved control and increased performance [12, 13]. Most of these model-based approaches found their way to practical applications and proved themselves satisfactory for a variety of complex systems [14]. Dynamic neural networks have been successfully exploited in fault detection for nonlinear systems [6-9].

In our attempt to study ANN-based control behaviour on turbine engines, we developed engine models in Simulink for various engines, such as the Bell 206 Jet Ranger helicopter Fig. 7, the Jet Cat RC engine Fig. 8 and other models in different stages of completion for various turbine engines at our disposal. Our dynamic models include the following major systems:

- Start sequence system Fig. 9 - controls the engine and ignition equipment in order to perform the ignition;
- Combustion chamber system - models the behaviour of gas dynamic and mechanical processes in the combustion chamber and power turbine;

- Starter NOR shutdown system - facilitates starter shutdown when the combustion chamber has a constant flame and regime that offers nominal speed;

- Virtual instrumentation system Fig. 10 - facilitates real-time monitoring of engine parameters, status and controls.

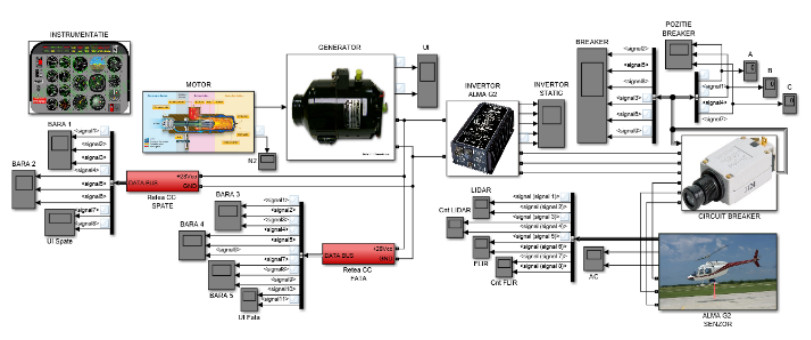

Fig. 7. Bell 206 Jet Ranger model blocks.

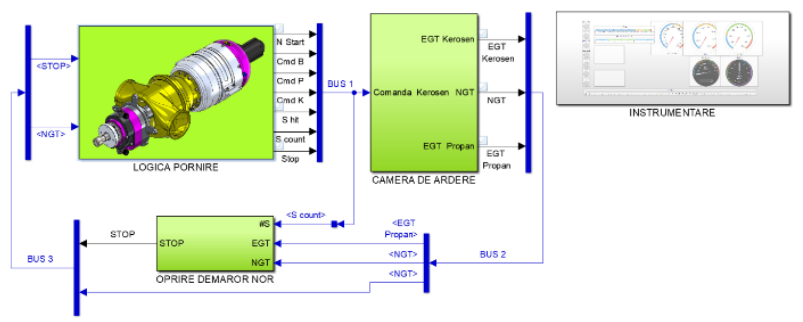

Fig. 8. Jet Cat SPT5 engine model blocks.

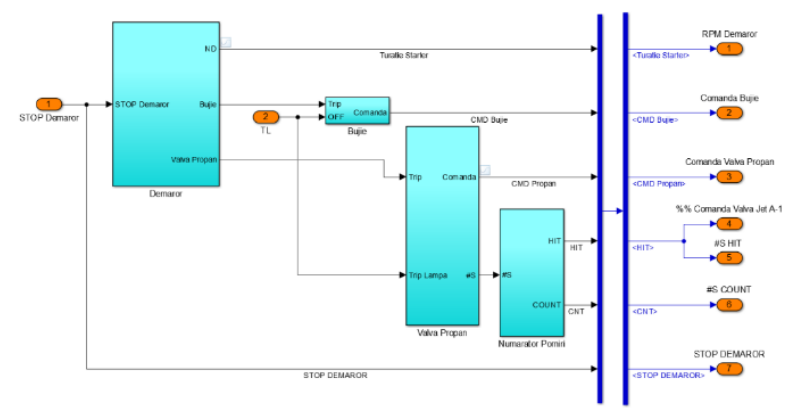

Fig. 9. Jet Cat SPT5 engine start sequence system.

Training these models showed smooth performance for the simulated engines, as showed in Fig. 11-14. A comprehensive power efficiency analysis is due to be showed with further studies.

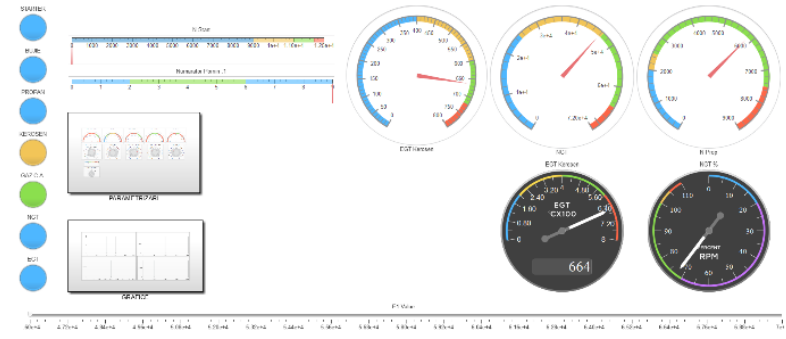

Fig. 10. Virtual instrumentation system. 


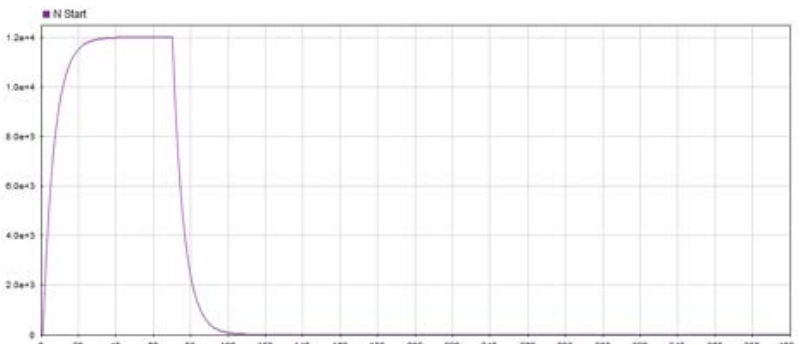

Fig. 11. Starter speed.

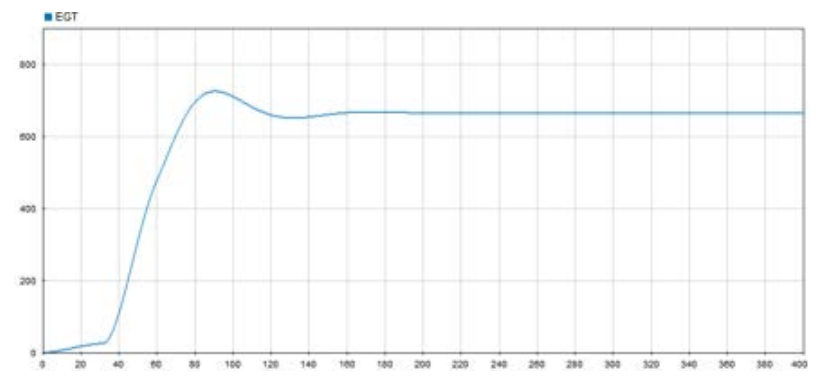

Fig. 12. Exhaust gas temperature (EGT).

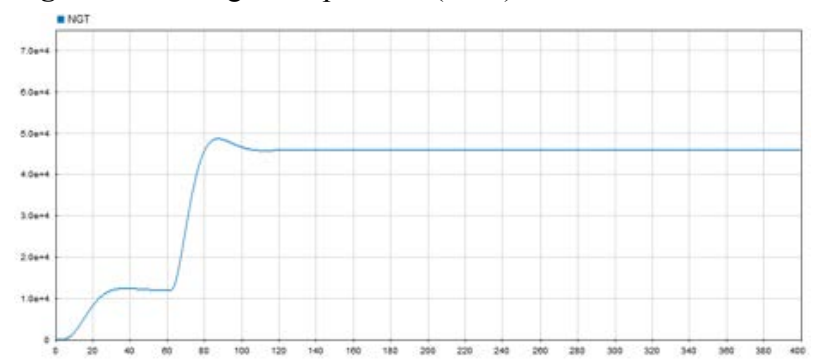

Fig. 13. Power turbine speed.

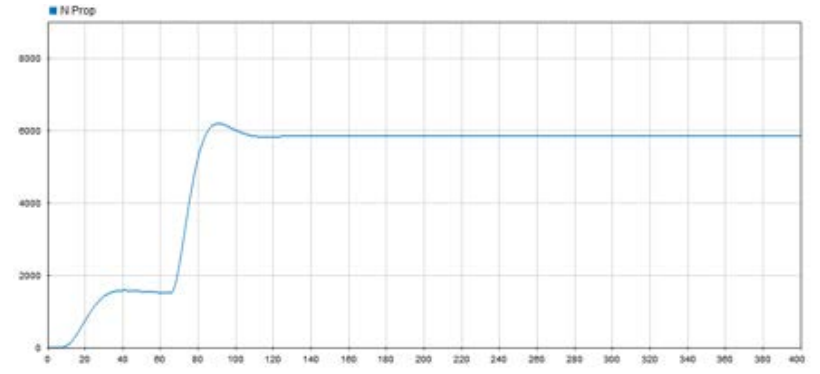

Fig. 14. Engine propeller speed.

In terms of instrumentation, fibre optics sensors are proposed instead of classic electrical sensors. These sensors bring several advantages: low power consumption, increased reliability, optimum parts and equipment dimensioning, sensor availability to hardlyaccessible or rush environments, high-speed data transmission, allowing instrumentation networks with multiple sensors connected in series. This technology has been successfully tested with aerospace engines, with results similar to thermocouples [15].

\section{APU test cell demonstrator}

To determine the capability of an adaptive intelligent control system to manage the power output we designed and built an experimental test rig contoured around a small turboprop engine. The characteristics of the small Jet Cat SPT5 engine are almost the same as in a medium sized APU. Temperatures, rpm`s and other characteristic parameters are around the same values, so is their gas dynamics and mechanical inertias. Given the fact that an APU uses a planetary or classic ratio reducing gearbox, the SPT5 model engine was a good platform to study the power output of the free power turbine. The compressor stage for the SPT5 is also comprised of a single stage impeller design with an air diffuser as seen in Fig. 15, like in most APU units Fig. 16.

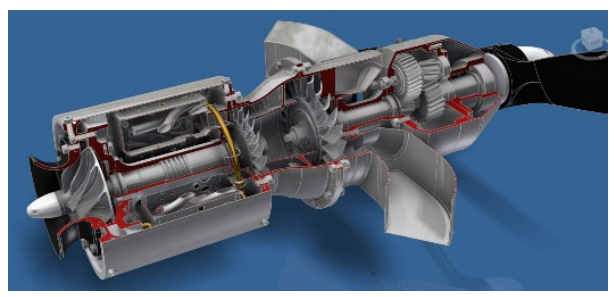

Fig. 15. Internal mechanics of a small turboprop engine similar to the SPT5 model.

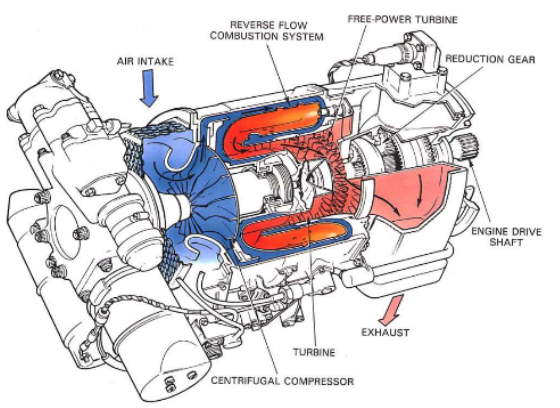

Fig. 16. Single stage compressor model of an APU unit.

Another common ground between the SPT5 and an APU is that they are both using a built-in starter engine. The main difference is comprised by the fact that the output of the APU is connected to a CSD (Constant Speed Drive) that in term is connected to an AC Electric Generator. Although they both have roughly the same output shaft $\mathrm{rpm}$ and torque. To mimic the resistive torque for the AC Generator of the APU we used a high pitch propeller, carbon honeycomb cell, with a maximum sustainable $18.000 \mathrm{rpm}$ as seen in Fig. 17.

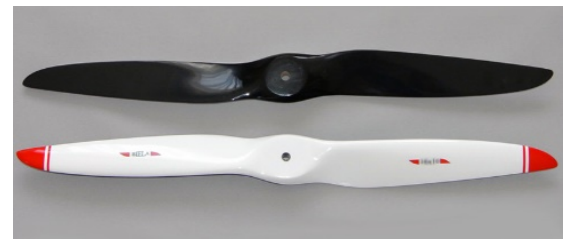

Fig. 17. Biella 30" x 10” 2 bladed propeller. 
In order to determine the engines, start ethic and control system we had to develop and build a test cell demonstrator as seen in Fig. 18.

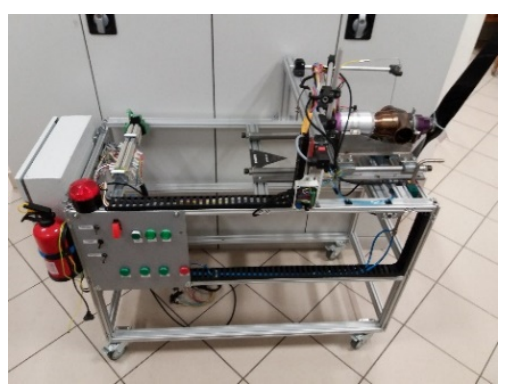

Fig. 18. Test cell demonstrator for micro engines and APU's.

The test stand is comprised of the following elements: stand skid fig. 18, engine mount with linear guide rails Fig. 19, instrumentation rail Fig. 20, operating panel Fig. 21, command electronics Fig. 22, acquisition rail with data bus Fig. 23, fuel system Fig. 24, load cell Fig. 25.

The test stand skid is built using $20 * 40 \mathrm{~mm}$ T-Slot extruded aluminium profiles assembled via interior threaded holes, M5 bolts, ' $\mathrm{L}$ ' fasteners and slotted channel M5 \& M3 T Nuts.

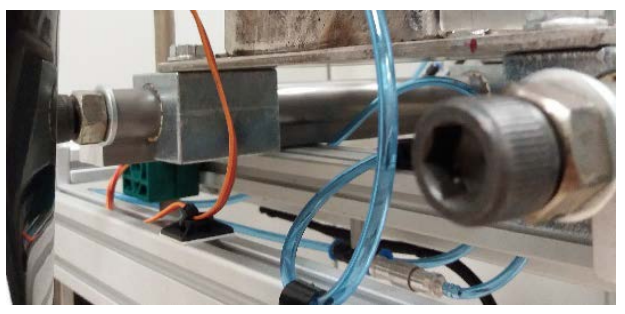

Fig. 19. Linear guide rails that hold the engine mount.

The instrumentation rail is comprised of sensor and instrument mounts which are adjustable to accommodate a variety of engines and sensor types.

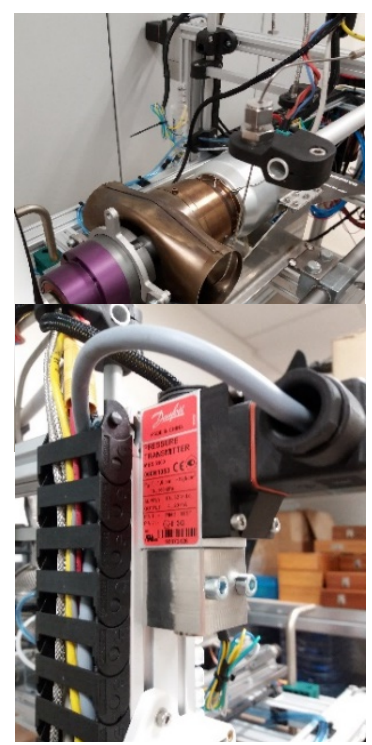

Fig. 20. Instrumentation rail \& instruments.

For safe control and operation, the test rig has a basic control panel with caution and warning beacons. This panel is used to isolate and inhibit or enable certain main systems of the test cell.

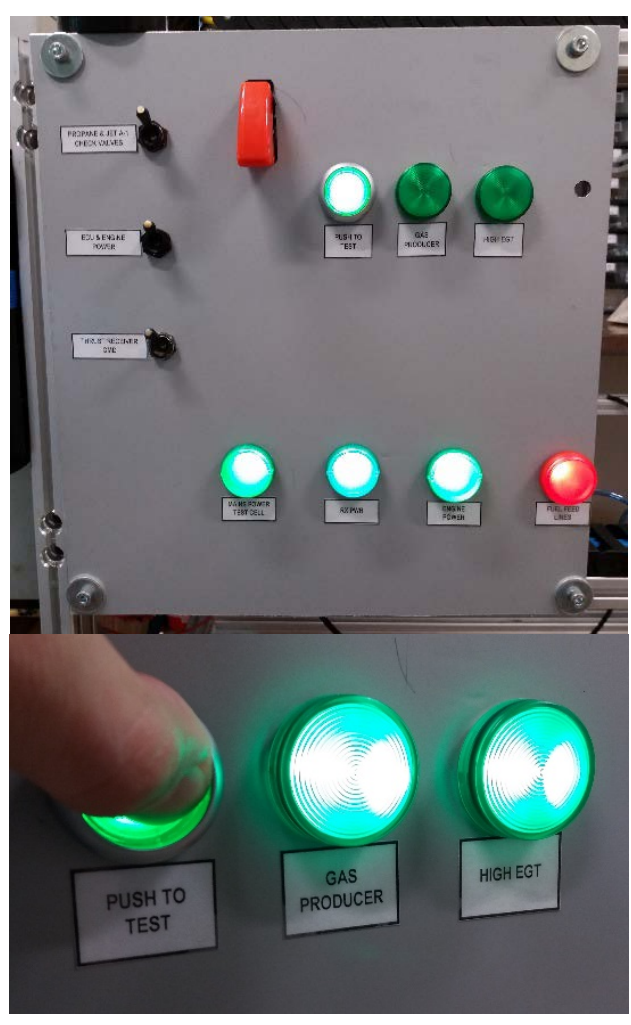

Fig. 20. Operating panel and caution beacons.

The command electronics bay is built around the engines original ECU with the addition of several protection, control and acquisition equipment used to monitor and control the test stand. 


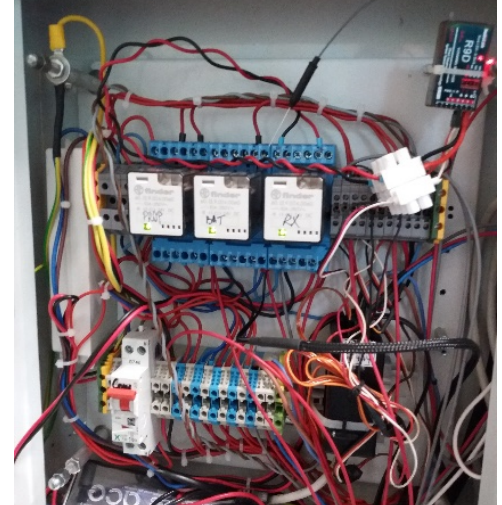

Fig. 22. Command bay electronics, back of test rig.

For developing a deterministic mathematical model of the turboprop engine, we used a system which is comprised of a data acquisition PLC (Programmable Logic Controller) from National Instruments. Signals are gathered from all sensors, instruments and systems in order to contour a dynamic model for the engine. These signals are fed into data collector rail and latterly fed thru a data bus comprised of a multiwire (26 line) cable.

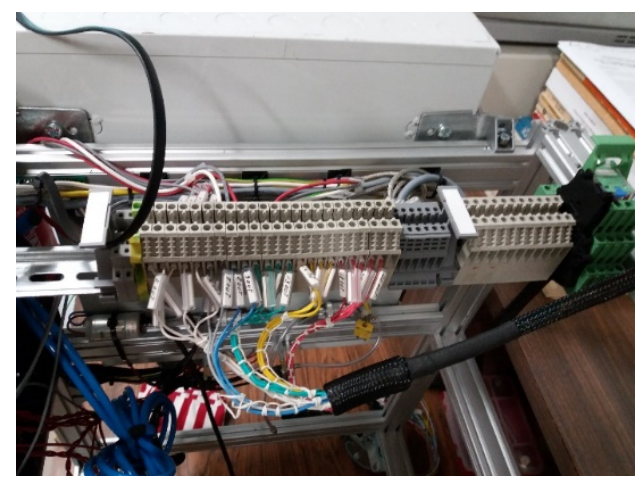

Fig. 23. Data rail feeders and data bus.

The Jet Cat engine uses a dual fuel system. One for engine start which uses high pressure propane gas and the other used for running the engine that uses JET A-1 fuel. All fuel lines, hydraulic and pneumatic use designated feed ports equipped with particle filters. Both lines (hydraulic and pneumatic) have installed solenoid actuated check-valves which are used for gas dosing (propane) and line isolation (propane $\&$ kerosene).

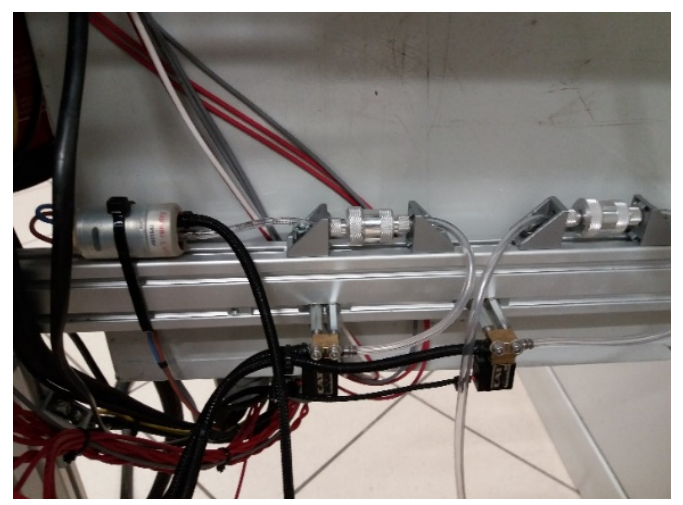

Fig. 24. Fuel system.
The load cell used is an $\mathrm{S}$ type compression cell with a maximum load capability of $100 \mathrm{~N}$.

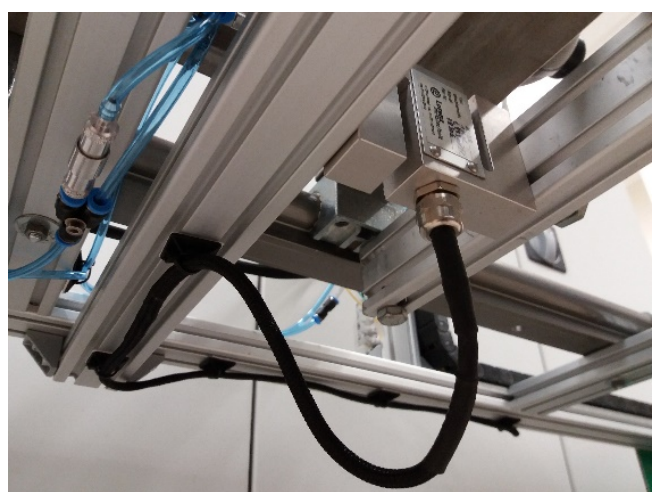

Fig. 25. Load cell and load beams.

\section{Dynamic model of the SPT5 engine}

After several experimental trials with the test stand for the SPT5 turboprop engine, we gathered a set of data strings and arrays regarding operating parameters and control ethics. Thus, we could contour the preliminary dynamic model, described in chapter 6 , tuned to the operation of the tested engine system. Also, we determined the propeller operational envelope in order to further calculate shaft power requirements for APU AC Generators.

In order to determine propeller performances, we used the formulas (1), (2) and (3) for dynamic thrust, static thrust and thrust vs. flight speed.

$$
\begin{gathered}
T_{d}=1.225 *\left[\pi *\left(k_{1} * D\right)^{\frac{1}{2}}\right] *\left[n * k_{1} * P *(1 / 60)\right]^{2} \\
-\left[n * k_{1} * P *(1 / 60) * V_{c}\right] *\left(\frac{D}{k_{2} * P}\right)^{1,5} \\
T_{S}=1.225 *\left[\pi *\left(k_{1} * D\right)^{\frac{1}{2}}\right] *\left[n * v * k_{1} * P *(1 / 60)\right]^{2} \\
-\left[n * v * k_{1} * P *(1 / 60)\right] *\left(\frac{D}{k_{2} * P}\right)^{1,5} \\
T_{R}=\rho *\left[\pi *\left(k_{1} * D\right)^{\frac{1}{2}}\right] *\left[n * k_{1} * P *(1 / 60)\right]^{2} \\
-\left[n * k_{1} * P *(1 / 60)\right] *\left(\frac{D}{k_{2} * P}\right)^{1,5}
\end{gathered}
$$

Where $\mathrm{D}$ is the propellers diameter in meters, $\mathrm{n}$ is speed in rpm, $\mathrm{P}$ is pitch in meters, $\mathrm{V}_{\mathrm{c}}$ is flight speed in $\mathrm{m} / \mathrm{s}, \rho$ is air density data vector in $\mathrm{kg} / \mathrm{m}^{3}, \mathrm{k}_{1}$ and $\mathrm{k}_{2}$ are propeller aerodynamic interference constants.

Given the above model we could plot graphs for static Fig. 25, Fig. 27, and dynamic thrust Fig. 28. 


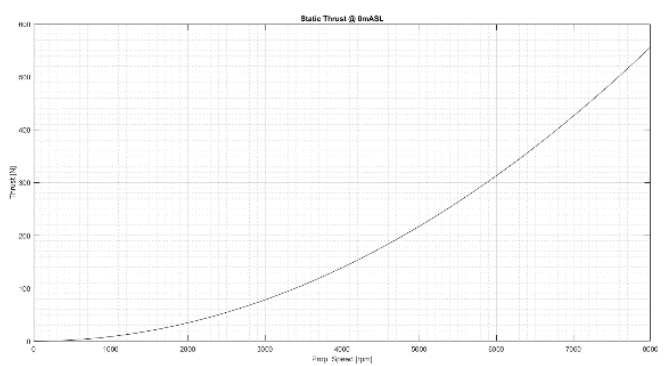

Fig. 25. Static thrust vs. propeller speed plot.

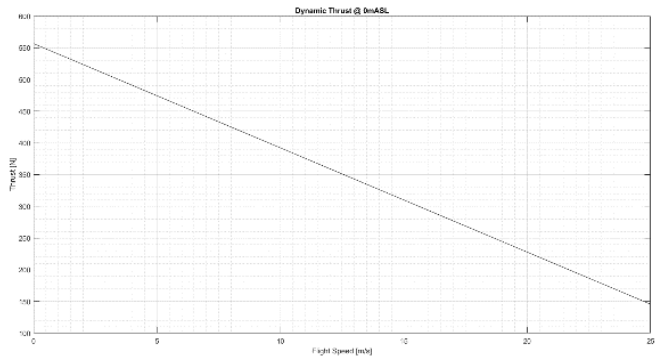

Fig. 26. Dynamic thrust vs. flight speed plot.

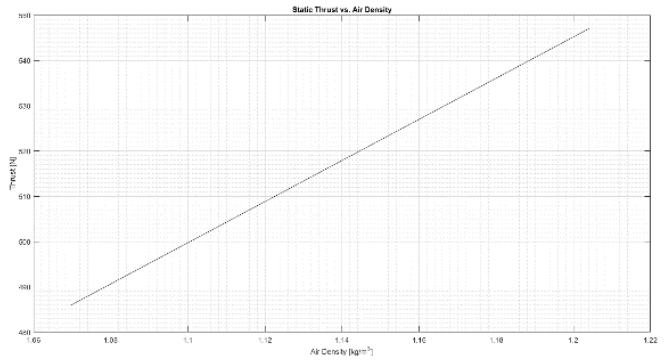

Fig. 27. Static thrust vs. air density plot.

Via means of data acquisition, we could determine the exact behaviour of the ECU's logic regarding the start sequence. For a detailed analysis we used a digital storage oscilloscope to measure the exact signal outputted from different systems, equipment and sensors.

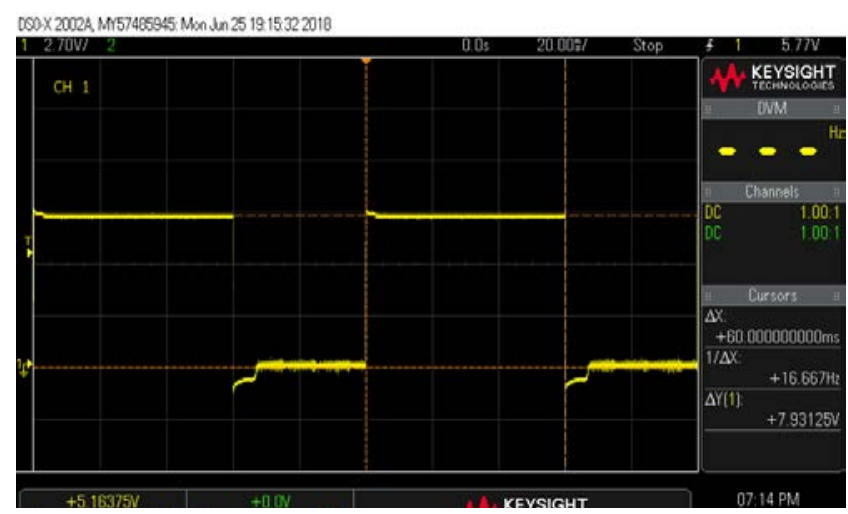

Fig. 28. Propane check-valve command signal.

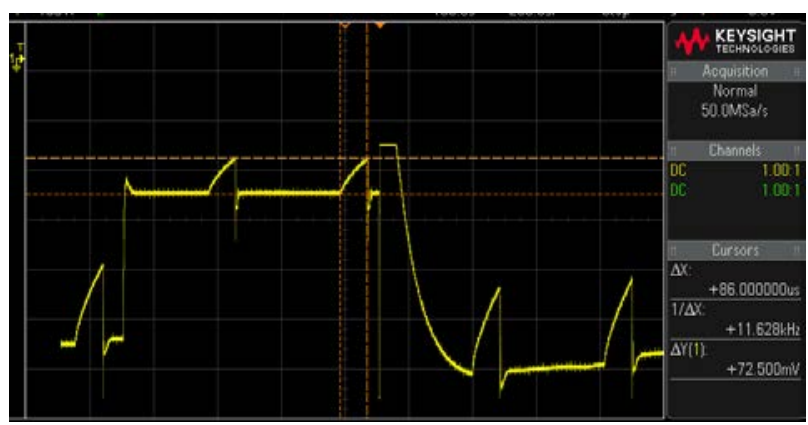

Fig. 29. Turbine rpm sensor`s digital signal

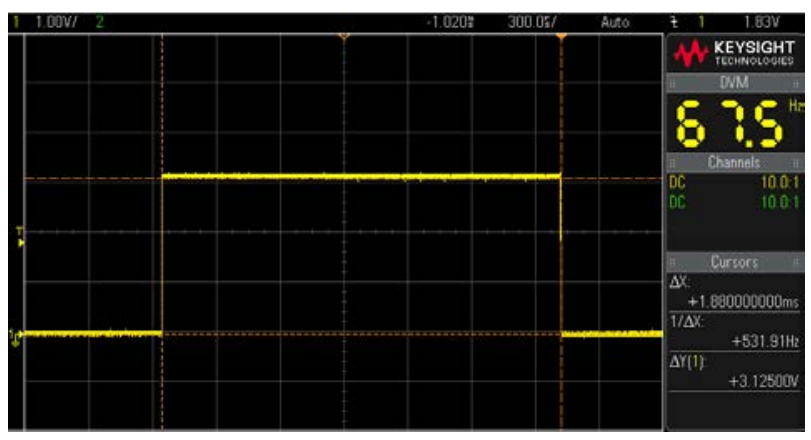

Fig. 30 PWM signal from throttle command at idle.

All subsystems in the Simulink model have built in transfer function in order to replicate the real system. For example, Fig. 31, the system EGT Jet A1 (Exhaust Gas Temperature of Kerosene) has the role to describe the gas dynamics of the combustion chamber when kerosene type fuel is injected. The block is comprised up of several subsystems which are designed to ensure fuel dosage to temperature dependency according to kerosene burn temperature, ambient temperature impact on the gases and several conditioning blocks used to activate or deactivate different systems and/or lamps in the instrumentation block.

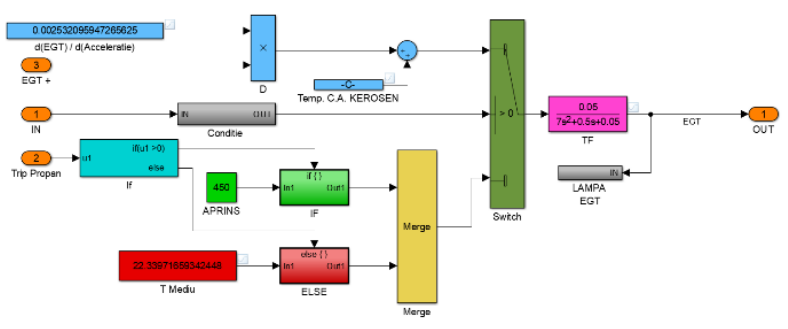

Fig. 31. Combustion chamber model.

The transfer function determined for the combustion chamber temperature variation is as in formula (4).

$$
H_{\text {EGT Jet } A 1}=\frac{0.05}{7 s^{2}+0.5 s+0.05}
$$

With the means of implementing transfer function we were able to duplicate as a mathematical model the exact temperature ramp of the combustion chamber.

The starter for the SPT5 engine is equipped with an solenoid actuated clutch designed to form a friction type 
coupling between the starter and the nose cone of the compressors impeller. The actuating logic of this system and the starter's dynamic model is illustrated in Fig. 32, respectively Fig. 33.

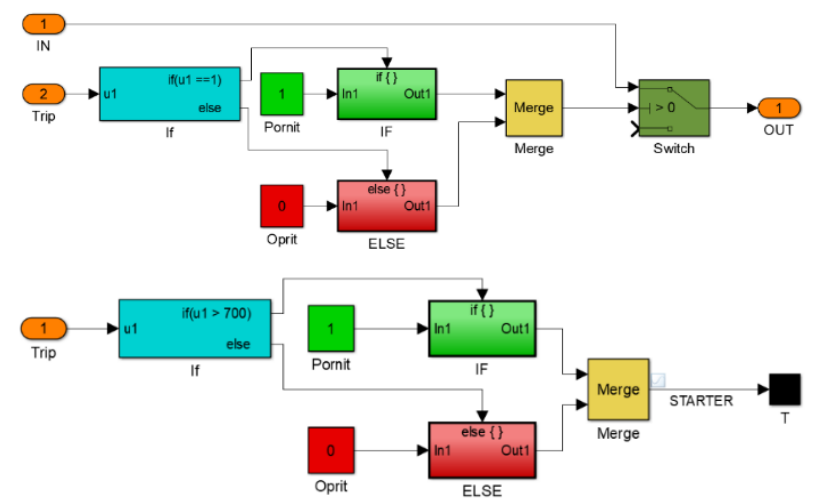

Fig. 32. Starter clutch operation logic.

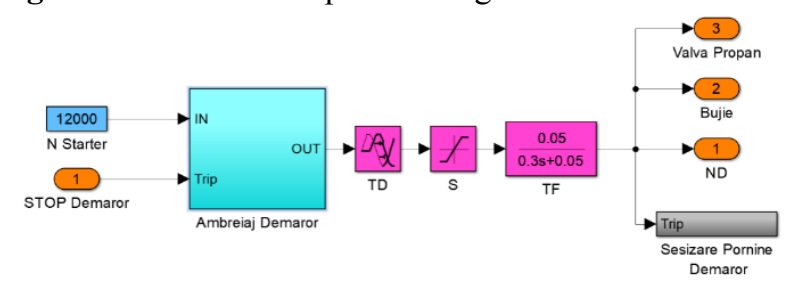

Fig. 33. DC Electric Starter motor dynamic model.

The transfer function for the starter motor is described in formula (5).

$$
H_{\text {starter }}=\frac{0.05}{0.3 s+0.05}
$$

The turboprop engine used, unlike APU`s, utilises a glow plug instead of a spark plug. This is mainly due to the fact that the SPT5 engine uses propane gas a starter fluid instead of a liquid fuel. The glow plug`s dynamic model is illustrated in Fig. 34, and it's transfer function in formula (6).

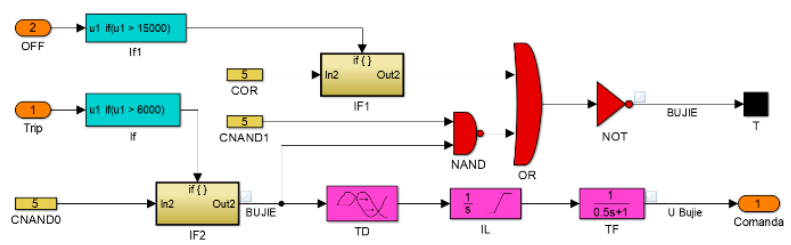

Fig. 34. Glow plug igniter dynamic model.

$$
H_{g p i}=\frac{1}{0.5 s+1}(6)
$$

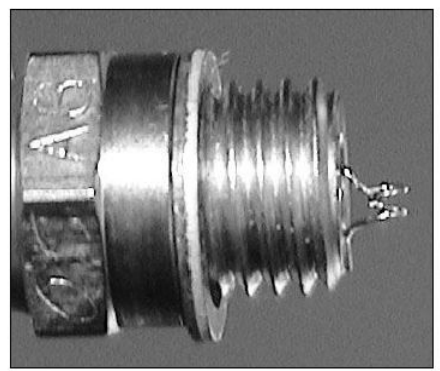

Fig. 35. Glow plug igniter.
For dosing starter gas, the SPT5 engine uses a solenoid actuated check-valve in order to feed propane gas to the combustion chamber. This check-valve operates intermittently in surges of 3 seconds at an actuating frequency of $16.66 \mathrm{~Hz}$. The mathematical system is described in Fig. 36.

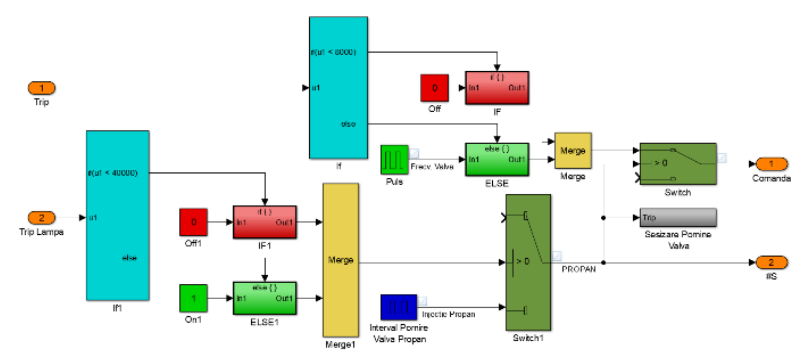

Fig. 36. Propane dosage check-valve.

Due to the use of propane as a starter gas it was necessary to determine the behaviour of the combustion chamber when fed with starter gas. Thus, the system EGT Gas emerged as illustrated in Fig. 37, with the corresponding transfer function (7).

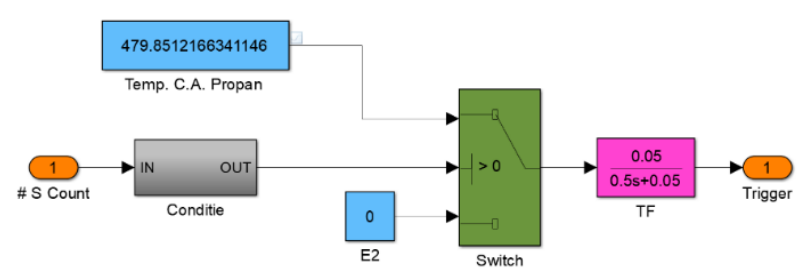

Fig. 37. EGT Gas system block.

$$
H_{\text {EGT Gas }}=\frac{0.05}{0.05 s+0.05}(7)
$$

After the ECU senses flame presence, via the built-in type $\mathrm{K}$ thermocouple in the combustion chamber, it monitors it's consistency and if it is constant, it feeds into the combustion chamber kerosene. This logic system is presented in Fig. 38.

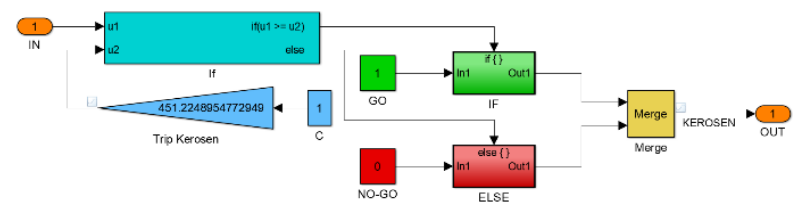

Fig. 38. Kerosene check-valve command system.

As in a conventional APU, the SPT5 turboprop engine, is equipped with a free power turbine. To replicate it's dynamic model we used the system in Fig. 39 along with the transfer function (8).

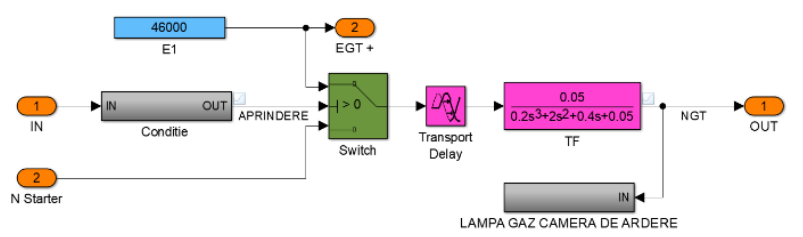


Fig. 39. Free power turbine model.

$$
H_{\text {turbine }}=\frac{0.05}{0.2 s^{3}+2 s^{2}+0.4 s+0.05}
$$

For the modelling of the gear ratio we used the system in Fig. 40. Note the transport delay block - it is used to replicate the resistive torque in the load bearing of the turbine and the resistive torque of the gearbox.

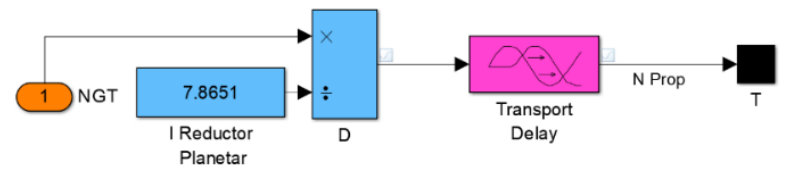

Fig. 40. Gearbox system modelling.

After the engine has an autonomous self-sustained combustion via the means of propane and kerosene fuel mixture it is necessary to disengage the starter motor. This is achieved only when certain parameters satisfy a know criteria. Thus, the model in Fig. 41. is used to do such that.

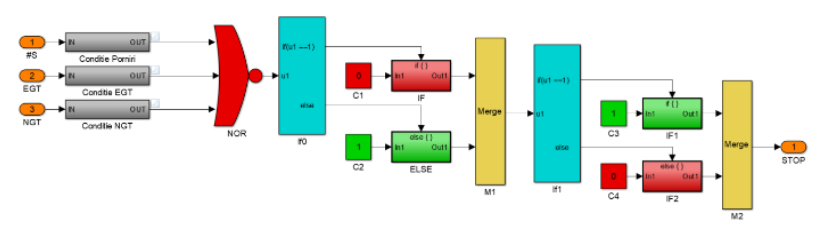

Fig. 41. Starter motor \& clutch disengage logic.

Due to the use of propane as a starting gas and of the glow plug igniter, there is a chance that the engine might not be able to start during the firs injection of gas. Thus, only after a minimum of 8 propane injection start attempts are the starter engine and glow plug igniter disengaged. For this to be achieved it's necessary to implement a propane injection counter in a conditioning system. The start manoeuvre will be aborted if after 8 propane gas injections the gas temperature in the combustion chamber is bellow $100^{\circ} \mathrm{C}$. This system is illustrated in Fig. 42.

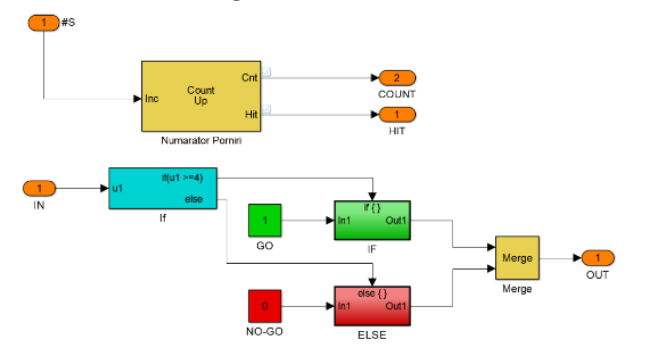

Fig. 42. Propane gas injection counter block.

\section{Conclusion}

Given the dynamic model developed via the means of experimental observation it is possible to conclude that there are possible means of reducing start times, idle ramps and fuel consumption in APU's. Due to the complexity of the system we decided to undergo development on both a neural network adaptive controller and also on a fuzzy logic controller to compare both in means of optimums. Through the use of this neural network-based system, it is possible to control the APU in such a way that it will have minimum energy loss during regime transit, minimum fuel burn throughout flight stages and thus expanding the resource of the unit (APU), given minimum stress as a result of stable change rates. The use of a PID based controller is also possible but without a significant fuel burn reduction. Also, we can stipulate that the APU will not have a smooth operation, instead it will have a significant variation in the command curve. Our ambition is to see that the neural network-based controller can successfully and optimally control the APU with the least amount of fuel burnt and the most output power possible. To further improve and validate a full reliable and operational test engine, we will be using by the end of 2018, early 2019, a HIL (Hardware in The Loop) testing system. This will allow to safely train any type of intelligent control system that we might use to control the engine. As a consequence, to using a HIL based validation system, it is possible to obtain high levels of performance in the means of fuel economy, thus reducing gas emissions, and furthermore of increasing the life resource of the engine and it's mechanics. The prolonging of exploitation resource is due to the fact that start times are reduced and more importantly, over speeding of the turbines will be impossible due to command signals that lack over shots in amplitude. There for, maintenance costs will be reduced in terms of worn part and components.

\section{References}

1. Rolls-Royce plc, 1996; The Jet Engine; Fifth Edition, ISBN 0902121235

2. Kourosh R., Andrew Gray, Neural Network Based Model Reference Controller for Active Queue Management of TCP Flows, Jet Propulsion Laboratory, California Institute of Technology, Pasadema CA91109, IEEEAC paper \#1408, Version 3, $22^{\text {nd }}$ Nov. 2004.

3. Simond Haykin, Kalman Filtering and Neural Networks, ISBN 0-471-22154-6, Canada 2002.

4. A. Eid, M. Abdel-Salam, H. El-Kishky, T. El-Mohandes, Simulation and transient analysis of conventional and advanced aircraft electric power systems with harmonics mitigation, Electric Power Systems Research, 79, 4, pp. 660-668 (2009)

5. J. S. Ra, A. K. Al-Suiada, O. Y. M. Al-Rawi, Power Distribution Systems 
Reconfiguration Bases on Artificial Neural Network and Genetic Algorithm for Loss Reduction, IFRSA International Journal of Electronics Circuits and Systems, 2, 1, pp. 26-36 (2013)

6. Glenn D. Schilling, Modelling Aircraft Fuel Consumption with a Neural Network, M.Sc. Thesis, The Faculty of the Virginia Polytechnic Institute and State University, Blacksburg, Virginia, US (1997)

7. "Intelligent Flight Control System", NASA Facts, Dryden Flight Research Centre (2002)

8. Anthony J. Calise, Rolf T. Rysdyk, Nonlinear Adaptive Flight Control using Neural Networks, IEEE Control System Magazine, 18, 6, pp. 14-25 (1998)

9. S. S. Tayarani-Bathaie, Z.N. Sadough Vanini, K. Khorasani, Dynamic neural network-based fault diagnosis of gas turbine engines, Neurocomputing 125 , pp. 153-165 (2014)

10.l. Al-Dein Al Zyoud, K. Khorasani, Neural Network-based Actuator Fault Diagnosis for Attitude Control Subsystem of a Satellite, World Automation Congress, Budapest, Hungary (2006)

11. A. Valdes, K. Khorasani, L. Ma, Dynamic Neural Network-Based Fault Detection and Isolation for Thrusters in Formation Flying of Satellites, Advances in Neural Networks - ISSN 2009, Lecture Notes in Computer Science, 553, Springer, Berlin, Heidelberg, pp. 780-793 (2009)

12. R. Mohammadi, E. Naderi, K. Khorasani, S. Hashtrudi-Zad, Fault Diagnosis of Gas Turbine Engines by Using Dynamic Neural Networks, ASME Turbo Expo 2010: Power for Land, Sea, and Air, 3, Glasgow, UK (2010)

13. A. J. Chipperfield, B. Bica, P. J. Fleming, Fuzzy scheduling control of a gas turbine aero-engine: a multiobjective approach, IEEE Transactions on Industrial Electronics, 49, 3, pp. 536$548(2002)$
14.A. Zilouchian, M. Juliano, T. Healy, J. Davis, Design of a fuzzy logic controller for a jet engine fuel system, Control Engineering Practice, 8, 8, pp. 873-883 (2000)

15.L. C. Jaw, S. Garg, Propulsion Control Technology Development in the United States (A Historical Perspective), NASA Glenn Research Center, Cleveland, Ohio, USA (2005)

16. J. S. Litt, D. L. Simon, S. Garg, A Survey of Intelligent Control and Health Management Technologies for Aircraft Propulsion Systems, NASA Glenn Research Center, Cleveland, Ohio, USA (2005)

17.P. J. Antsaklis, K. M. Passino, An Introduction to Intelligent and Autonomous Control, Kluwer Academic, Boston, Mass, USA (1993)

18. M. Pakmehr, B. Moslehi, J. Costa, R. J. Black, V. Sotoudeh, A. R. Behbahani, A Review of Fibre Optic Networks for Turbine Engine Instrumentation Channel: Control, PHM, and Test Cell Applications, $5^{\text {th }}$ AIAA/ ASME/ SAE/ ASEE Joint Propulsion Conference, Cleveland $\mathrm{OH}(2014)$ 